\title{
Epidemiology and Antimicrobial Resistance Patterns in Enteric Fever among patients in Garissa County, a Semi-Arid Region of North Eastern Kenya
}

\author{
Khatra Shariff Said ${ }^{1}$, Zipporah Ng'ang'a ${ }^{1}$ and Samuel Kariuki ${ }^{2}$ \\ ${ }^{1}$ Institute of Tropical Medicine and Infectious Diseases, Jomo Kenyatta University of Agriculture and \\ Technology, Nairobi, Kenya. \\ ${ }^{2}$ Centre for Microbiology Research, Kenya Medical Research Institute, Nairobi, Kenya.
}

\begin{abstract}
Typhoid and paratyphoid fever continue to be important causes of illness and death, particularly among children and adolescents in developing countries where enteric fever is associated with poor sanitation and unsafe food and water. Quantification of disease burden is crucial for policy making about the deployment of enteric fever prevention measures and vaccines. This cross-sectional study was undertaken to determine the epidemiology and antimicrobial resistance pattern in bacterial aetiologies of enteric fever among patients attending Garissa County Referral Hospital, (GCRH) located in a semi-arid region of North Eastern Kenya. Blood and stool samples were obtained from 379 consenting patients and a detailed sociodemographic questionnaire was administered. Isolation and identification of Salmonella Typhi, S. Paratyphi A and $S$. Paratyphi B were obtained by convectional culture, PCR and Vitek-2 compact detection method. Antimicrobial susceptibility testing was done using Kirby-Bauer's disc diffusion method. Multidrug resistance was defined as co-resistance to ampicillin, chloramphenicol and cotrimoxazole. Eight of the 379 (2.1\%) participants were positive for Salmonella spp. Of the 8 Salmonella isolates were $S$. Typhi $(\mathrm{n}=2 ; 25 \%), S$. Paratyphi A $(\mathrm{n}=2 ; 25 \%)$ and $S$. Paratyphi B $(\mathrm{n}=4 ; 50 \%)$. Resistance to ampicillin, tetracycline, gentamycin, chloramphenicol, nalidixic acid and trimethoprim-sulfamethoxazole was $100 \%, 87.5 \%$, $75 \%, 50 \%, 25 \%$ and $25 \%$ respectively. No isolate showed resistance to ciprofloxacin. Half of all $S$. typhi, S. paratyphi $A$ and B were multidrug-resistant. Risk factors including water and food (such as often eating outside homestead, family eating from a common plate, taking locally prepared cold drinks, family wash hands in common basin), low socio-economic status and availability of a previous laboratory confirmation of typhoid fever were associated with $S$. Typhi and $S$. Paratyphi infection. The isolation of a large proportion of MDR $S$. Typhi, $S$. Paratyphi $A$ and $\mathrm{B}$ is worrying. Although these isolates were susceptible to fluoroquinolones, there is need for routine surveillance to monitor susceptibility to the initial first line antibiotics in clinical settings since the MDR strains have lately shown increased resistance. Addressing issues of contaminated food, water, sanitation and hygiene and low socio-economic status is likely to prevent and reduce the burden on enteric fever in this region.
\end{abstract} Keywords: Enteric Fever, Molecular Epidemiology, Antimicrobial Resistance Pattern, Semi-Arid Region of North Eastern Kenya

DOI: $10.7176 / \mathrm{JHMN} / 60-14$

Publication date:March $31^{\text {st }} 2019$

\section{INTRODUCTION}

The genus Salmonella comprises Salmonella enterica and Salmonella bongori. Salmonella enterica subspecies I includes nearly all pathogenic serotypes for humans (Pegues and Miller, 2015). The growth of S. enterica Typhi and S. enterica Paratyphi A, B, and C is limited to humans, and these organisms cause enteric fever (Pegues and Miller, 2015). Non-typhoidal Salmonella (NTS) cause acute gastroenteritis - salmonellosis - a foodborne disease that is prevalent worldwide (Harris and Brooks, 2013; Pegues and Miller, 2015). Enteric-fever is one of the major public health problems in developing countries including Kenya (Radhakrishnan et al., 2018). Globally, 14.3 million cases of typhoid and paratyphoid fevers occurred in 2017 with a case fatality estimates of 135.9 thousand (GBD 2017 Typhoid and Paratyphoid Collaborators, 2019). The incidence of enteric-fever ranges from 100 to 199.9 per 100,000 population in Kenya (Radhakrishnan et al., 2018), but these figures may be underestimates due to under re porting, as only severely ill patients seek treatment in hospitals (Breiman et al., 2012)

The risk of enteric fever is highest in infants, young children and young adults with underlying comorbidities, including severe anaemia, malaria, malnutrition and HIV infection (Whitaker et al., 2009). The case fatality rate is high in those with HIV infection and among those living in lower-income countries due to overpopulation and poor hygiene (Whitaker et al., 2009; Ao et al., 2010; WHO, 2018). 
Problems are also emerging with the clinical treatment of typhoid in resource-poor settings. For many years, the antibiotics chloramphenicol, ampicillin, and cotrimoxazole formed the mainstays of typhoid treatment. In the past two decades, multidrug-resistant (MDR) strains of S. enterica have emerged worldwide (Phan et al., 2009). Reduced susceptibility to fluoroquinolones has been reported in Kenya (Kariuki et al., 2010). Changing trends in antibiotic resistance among $S$. enterica has appeared against ampicillin, co-trimoxazole, and chloramphenicol have also been reported in Kenya (Kariuki et al., 2006; Kariuki et al., 2010). Understanding local and regional antimicrobial susceptibility trends is vital in guiding empiric therapy. Monitoring and reporting of antimicrobial susceptibility can guide public health decision-making on the need for control strategies including vaccination.

In Kenya, MDR $S$. Typhi isolates from adults and school age children associated with sporadic outbreaks in resource-poor settings, especially in slum areas, have been reported (Kariuki et al., 2004). Unfortunately, data are scarce on the epidemiology and antimicrobial resistance pattern of $S$. Typh $i$ and $S$. Paratyphi species in Garissa County. This geographical region is marked by lack of one or more of the following five amenities: access to improved water, access to sanitation, durable housing, sufficient living area, and secure tenure (UN-HABITAT, 2006) essentially presenting risk factors for transmission of $S$. Typhi and Paratyphi occurs through consumption of contaminated food or water via short-cycle or long-cycle transmission. Evidence from studies in Kenya, India, Egypt, and Bangladesh demonstrate that morbidity and mortality in such areas are much higher than the national averages (Kimani-Murage et al., 2014; Bassiahi et al., 2014). Against this backdrop, this study was undertaken to determine the epidemiology and antimicrobial Resistance Patterns of $S$. enterica serovars in cases of clinically suspected enteric fever among patients in Garissa County, a Semi-Arid Region of North Eastern Kenya

\section{Materials and Methods \\ Study setting and design}

This was a descriptive cross-sectional study design conducted between March and December, 2018 consented adult population attending Garissa Provincial General Hospital. Applying the formula for estimating the population proportion with specified relative precision described by Lemeshow et al (1990) setting the $\alpha$ at 0.05 , and a detection rate of $50 \%$, a total of 379 patients were recruited to achieve 0.95 power. Inclusion criteria included presenting with fever $\left(38^{\circ} \mathrm{C}\right.$ and above) lasting for at least three days and accompanied with either of the following symptoms; abdominal pain, vomiting, diarrhea, constipation, headache, weakness, arthralgia or poor response to antimalarial medications. Participants were subjected to a face to face interview and also provided $10 \mathrm{ml}$ of venous blood and a loopful stool samples for culture. The samples were processed at Garissa County Referral hospital laboratory and only positive blood and stool specimens were characterized further for pathogenic Salmonella strains at Centre for Microbiology Research (CMR); Kenya Medical Research Institute in Nairobi. This study was approved by Ethical Review Committee of Kenya Medical Research Institute (KEMRI/SSC No.2464).

\section{Blood isolation}

The blood was cultured in broth media containing brain heart infusion and para-aminobenzoic acid, incubated at $37^{\circ} \mathrm{C}$, and subcultured when turbid onto sheep blood agar and MacConkey plates.

\section{Stool Culture}

All stool samples were placed in Cary Blair media and then plated into MacConkey (MAC) and Deoxycholate Citrate Agar (DCA). Portions of whole stool were also placed into Selenite -F broth for subculture and incubated at $37^{\circ} \mathrm{C}$ overnight. A subculture of Selenite broth on Mac Conkey agar, and xylose-lysine deoxycholate agar were made from the surface of the broth without disturbing the sediment. The plates were incubated at $37^{\circ} \mathrm{C}$ for $18-24$ hours.

All suspected Salmonella colonies were picked from the agar plates subjected to biochemical tests, PCR amplification of invA gene and subsequently serotyped using the VITEK 2 system, Version 0.8.01 (bioMe' rieux, Inc., Hazelwood, MO).

\section{Biochemical Tests}

All suspected Salmonella colonies were picked from the agar plates and inoculated into the following biochemical test tubes for confirmation as described by Kebede et al., (2016): triple sugar iron (TSI) test (presumptive Salmonella colonies produce black colonies or colonies with black centers and red medium on TSI agar) (OXOID, England), citrate test (presumptive Salmonella colonies produce blue color for the citrate test), urease test (presumptive Salmonella colonies produce purple-red color for the urease test), lysine decarboxylase (LDC) agar (OXOID, England) test (presumptive Salmonella colonies produce purple-colored colonies on LDC agar), and 
indole test (presumptive Salmonella colonies produce violet-colored colonies for the indole test). Plates were incubated for 24 or $48 \mathrm{hrs}$ at $37^{\circ} \mathrm{C}$. Colonies were also tested for catalase production

\section{PCR detection of Salmonella spp}

The boiling method was used to extract Salmonella plasmid or DNA template. Briefly, a single bacterial colony was picked from the Luria-Bertani (LB) agar plate, boiled in $50 \mu \mathrm{l}$ distilled water for 10 min and immediately cooled on ice for $5 \mathrm{~min}$. After a short spin, $4 \mu \mathrm{l}$ of this solution was used as PCR template as described by Kebede et al., (2016). The bacterial DNA template was amplified using $0.5 \mu \mathrm{M}$ primers specific for invA gene comprising of: forward primer GAG GAA GGG AAATGA AGC TTT T and reverse primer TAG CAA ACT GTCTCC CAC CAT AC, PCR buffer [10 mM Tris/ $\mathrm{HCl}(\mathrm{pH} 8,3), 50 \mathrm{mM} \mathrm{KCl}, 3 \mathrm{mM} \mathrm{MgCl}$, and $0.01 \%$ gelatin], $200 \mu \mathrm{M}$ of each dNTP, and 1.0 U AmpliTaq Gold enzyme (Roche Molecular Systems, Inc, Brachburg, New Jersey, USA). The mixtures were amplified under the following cycling condition: 40 cycles at $94^{\circ} \mathrm{C}$ for 1 minute, $55^{\circ} \mathrm{C}$ for 1 minute, and $72^{\circ} \mathrm{C}$ for 2 minutes, with a final extension at $72^{\circ} \mathrm{C}$ for 10 minutes in an automated thermal cycler (Control system PC- 710; Astec; Tokyo, Japan). An aliquot of $10 \mu \mathrm{l}$ of each amplified product was visualized in $2 \%$ (wt/vol) agarose gel electrophoresis.

\section{Serotyping}

The bacterial isolates positive by the genus-specific PCR were serotyped by slide agglutination test targeting specific flagellar antigens. Further, serotyping was done using the VITEK 2 system, Version 0.8.01 (bioMe rieux, Inc., Hazelwood, MO) according to the manufacturers instruction.

\section{Antimicrobial susceptibility testing}

Each isolate was tested for susceptibility to antimicrobials by a controlled disk diffusion technique on Diagnostic Sensitivity Testing (DST) agar (Oxoid Ltd., Basingstoke, United Kingdom) plates containing 5\% lysed horse blood. Salmonella isolates were tested for susceptibility to the following 7 antibiotics (OXOID, England): ampicillin $(10 \mu \mathrm{g})$, tetracycline $(30 \mu \mathrm{g})$, trimethoprim/sulfamethoxazole $(30 \mu \mathrm{g})$, chloramphenicol $(30 \mu \mathrm{g})$, gentamicin $(10 \mu \mathrm{g})$, ciprofloxacin $(5 \mu \mathrm{g})$, and nalidixic acid $(30 \mu \mathrm{g})$ using the disk diffusion method according to guidelines set by the Clinical Laboratory Standards Institute (CLSI, 2017). Antibiotic impregnated discs were dispensed on the surface of cultures of Muller-Hinton agar and incubated at $35^{\circ} \mathrm{C}$ for $20 \mathrm{hrs}$. The diameters of the zones of inhibition were recorded to the nearest $\mathrm{mm}$ and classified as resistant, intermediate, or susceptible according to established interpretive chart (CLSI, 2017). Escherichia coli ATCC 25922 was used as a reference strain in the disk diffusion susceptibility tests.

\section{Data Analysis}

Frequency (\%), mean and standard deviation, were used to describe the qualitative and laboratory parameters. Chisquare or Fisher's exact test were used to test for significance where applicable. In bivariate analyses, odds ratios (OR) and 95\% confidence intervals (CI) for the association between enteric fever infection and socio-demographic, hygienic and environmental characteristics were calculated using Poisson regression. In multivariate analyses, a manual backward elimination approach was used to reach the most parsimonious model, including factors that were associated with infection of enteric fever at the significance level of P 0.05. All statistical analyses were performed using STATA v 13 (StataCorp LP, College Station, TX, USA).

\section{RESULTS}

\section{Baseline characteristics}

Analyzable data were available for all the 379 participants recruited. The mean ( \pm standard deviation - SD) age of the participants was 37.3 ( \pm 13.3 ) years ranging (18 to 95 years). The majority of the participants $39.8 \%$ were aged 18- 30 years, $54.4 \%$ were female, $58 \%$ married, $39.3 \%$ had secondary level education, $45.1 \%$ were in business for their occupation. Table 1 summarizes the characteristics of the study population. Further, the majority of participants $67.3 \%$ had household population of 1 to 5 people, $16.6 \%$ kept chicken, $67.5 \%$ had $<20,000.00 \mathrm{kshs}$ monthly income, $75.2 \%$ had body temperature $>37.1^{\circ} \mathrm{C}$, while $58.8 \%$ had headache. About $9.8 \%$ of the participants reported receiving treatment, only $7.4 \%$ had laboratory confirmation of typhoid while $35.1 \%$ had history of typhoid of fever. Only $10 \%$ had history of contact with typhoid patient in the last one year, $20.1 \%$ often eat outside with $19.8 \%$ eating from common plate while $13.2 \%$ who took locally prepared cold drinks. The majority $91.8 \%$ reported frequently washing their hands with $15 \%$ washing their hands in a common basin. The majority $72.8 \%$ obtained their drinking water from wells, $72.3 \%$ treated their drinking water and $67 \%$ had access to a modern toilet for the sanitation. 
Table 1: Baseline characteristics of study population

\begin{tabular}{|c|c|c|c|}
\hline Variables & Unit & Frequency & Percentage \\
\hline Gender & Female & 206 & 54.4 \\
\hline Age (Years) & $18-30$ & 151 & 39.8 \\
\hline Marital status & Married & 220 & 58 \\
\hline Education level & Secondary & 149 & 39.3 \\
\hline Occupation & Bussiness & 171 & 45.1 \\
\hline Religion & Muslim & 303 & 79.9 \\
\hline Household population & 1 to 5 & 255 & 67.3 \\
\hline Family owns cows & Yes & 71 & 18.7 \\
\hline Family owns goats & Yes & 88 & 23.2 \\
\hline Family owns chicken & Yes & 63 & 16.6 \\
\hline Monthly income (Kshs) & $<20,000.00$ & 256 & 67.5 \\
\hline House building material & Concrete & 230 & 60.7 \\
\hline Weight & $>51$ & 328 & 86.5 \\
\hline Body Temperature & $>37.1$ & 285 & 75.2 \\
\hline Days with Fever & $<3$ Days & 300 & 79.2 \\
\hline Signs and symptoms & Headache & 223 & 58.8 \\
\hline Receiving treatment & Yes & 37 & 9.8 \\
\hline Laboratory confirmation of typhoid & Yes & 28 & 7.4 \\
\hline History of typhoid fever & Yes & 133 & 35.1 \\
\hline \multicolumn{4}{|l|}{$\begin{array}{l}\text { Had contact (work colleagues, school mates, } \\
\text { relatives, neighbours) with typhoid patients within }\end{array}$} \\
\hline $\begin{array}{l}\text { one year before } \\
\text { Did vou travel outside vour community rece }\end{array}$ & Yes & 38 & 10 \\
\hline (three months ago)? & Yes & 31 & 8.2 \\
\hline Often eat from outside & Yes & 76 & 20.1 \\
\hline Family eat from a common plate & Yes & 75 & 19.8 \\
\hline Take locally prepared cold drinks & Yes & 50 & 13.2 \\
\hline Often wash your hands & Usually & 348 & 91.8 \\
\hline Family wash hands in common basin & Yes & 57 & 15 \\
\hline Sources of water supplies in the family & Wells & 276 & 72.8 \\
\hline Treat drinking water & Yes & 274 & 72.3 \\
\hline Method for water treatment & Chemical & 146 & 38.5 \\
\hline Method for food storage & Fridge & 38 & 10 \\
\hline Type of latrine do you have & Modern Toilet & 254 & 67 \\
\hline
\end{tabular}




\section{Molecular and Serotype Identification of Salmonella}

Among a total of 379 samples examined for bacteriological status, 8 participants were positive for Salmonella. The PCR amplifications gave products of $496 \mathrm{bp}$ for the 8 isolates, expected size for samples positive for Salmonella by the genus-specific PCR reaction. Serotyping also revealed the same 8 isolates to be Salmonella spp. Out of the 8 Salmonella positive strains $(\mathrm{n}=2 ; 25 \%)$ were Salmonella enterica subspecies enterica serovar typhi, $(\mathrm{n}=2 ; 25 \%)$ were Salmonella Paratyphi A with the majority $(\mathrm{n}=4 ; 50 \%)$ being Salmonella Paratyphi B.

\section{Antibiotic susceptibility profiles of Salmonella strains}

Single and multiple resistance to most of the antibiotics tested were observed. The highest prevalence of resistance observed was to ampicillin with all $8(100 \%)$ isolates being resistant. The next highest resistance was to Tetracycline, with $7 / 8$ isolates $(87.5 \%)$ being resistant and 1 being intermediate-resistant. There were $6 / 8(75 \%)$ isolates resistant to Gentamycin with two isolates 1(CMR_5722) and 2(CMR_6704) being susceptible and intermediate resistant respectively. Ciprofloxacin was the most effective antibiotics, all isolates $(100 \%)$ were sensitive. Nalidixic acid and Trimethoprim-sulfamethoxazole were the second most effective antibiotics, except that 1 (CMR_6772 and CMR_6704) and 2 (CMR_8548 and CMR_5732) isolates were resistant to the two antibiotics, respectively. Fifty percent (4/8) of the isolates were found to be susceptible to Chloramphenicol (Table 2).

Table 2. Antibiotic disk diffusion susceptibility test results for salmonella strains

\begin{tabular}{|c|c|c|c|c|c|c|c|c|}
\hline \multirow[t]{2}{*}{ Isolate } & \multirow[t]{2}{*}{ Strain } & \multicolumn{7}{|c|}{ Antibiotic susceptibility profiles } \\
\hline & & AMP & NA & CHLOR & GEN & CIP & SXT & TET \\
\hline CMR_8530 & S. paratyphi $A$ & $\mathrm{R}$ & $\mathrm{S}$ & $\mathrm{S}$ & $\mathrm{R}$ & $\mathrm{S}$ & $\mathrm{S}$ & $\mathrm{R}$ \\
\hline CMR_6772 & S. paratyphi B & $\mathrm{R}$ & $\mathrm{R}$ & $\mathrm{S}$ & $\mathrm{R}$ & $\mathrm{S}$ & $\mathrm{S}$ & $\mathrm{R}$ \\
\hline CMR_8548 & S. paratyphi $B$ & $\mathrm{R}$ & $\mathrm{S}$ & $\mathrm{R}$ & $\mathrm{R}$ & $\mathrm{S}$ & $\mathrm{R}$ & $\mathrm{R}$ \\
\hline CMR_8574 & S. paratyphi B & $\mathrm{R}$ & $\mathrm{S}$ & $\mathrm{S}$ & $\mathrm{R}$ & $\mathrm{S}$ & $\mathrm{S}$ & $\mathrm{R}$ \\
\hline CMR_6704 & S. paratyphi $A$ & $\mathrm{R}$ & $\mathrm{R}$ & $\mathrm{R}$ & I & $\mathrm{S}$ & $\mathrm{S}$ & $\mathrm{R}$ \\
\hline CMR_5792 & S. paratyphi $B$ & $\mathrm{R}$ & $\mathrm{S}$ & $\mathrm{R}$ & $\mathrm{R}$ & $\mathrm{S}$ & $\mathrm{S}$ & $\mathrm{R}$ \\
\hline CMR_5722 & S. enterica & $\mathrm{R}$ & $\mathrm{S}$ & $\mathrm{S}$ & $\mathrm{S}$ & $\mathrm{S}$ & $\mathrm{S}$ & I \\
\hline CMR_5732 & S. enterica & $\mathrm{R}$ & $\mathrm{S}$ & $\mathrm{R}$ & $\mathrm{R}$ & $\mathrm{S}$ & $\mathrm{R}$ & $\mathrm{R}$ \\
\hline
\end{tabular}

S - Susceptible; R - Resistant; I - Intermediate-resistant; AMP-Ampicillin; NA - Nalidixic acid; CHLOR - Chloramphenicol;

GEN - Gentamycin; CIP - Ciprofloxacin; SXT - Trimethoprim-sulfamethoxazole; TET - Tetracycline

\section{Multivariate analyses}

After adjusting for confounders, Laboratory confirmation of typhoid (OR 66.6, 95\% CI 5.8-757.2), often eating outside homestead (OR 5.3, 95\% CI 1.4-12.4), family eating from a common plate (OR 6.1, 95\% CI 1.2-21.2), taking locally prepared cold drinks (OR 6.9, 95\% CI 1.4-32.3), family wash hands in common basin (OR 7.3, 95\% CI 1.9-31.2) and the participants who had monthly income Kshs $<20,000.00(<200$ USD) (OR $0.2,95 \%$ CI 0.003 0.8 ) were independently associated with pathogenic salmonella infection. Table 3 summarizes the multivariate analysis of factors independently associated with enteric fever. 
Table 3. Adjusted factors associated with pathogenic salmonella infection

\begin{tabular}{|c|c|c|c|c|c|}
\hline \multirow{3}{*}{ Variables } & \multirow{3}{*}{ Total } & \multicolumn{2}{|c|}{ Pathogenic Salmonella } & \multirow{3}{*}{$P$ - value } & \multirow{3}{*}{$\begin{array}{c}\text { Multivariate } \\
\operatorname{aOR}(95 \% \mathrm{CI})\end{array}$} \\
\hline & & infe & tion & & \\
\hline & & Frequency & Percentage & & \\
\hline \multicolumn{6}{|c|}{ Laboratory confirmation of typhoid } \\
\hline Yes & 28 & 7 & 25 & 0.001 & $66.6(5.8-757.2)$ \\
\hline No & 351 & 1 & 0.3 & Reference & Reference \\
\hline \multicolumn{6}{|c|}{ Do you often eat from outside } \\
\hline Yes & 76 & 5 & 6.6 & $\mathbf{0 . 0 3}$ & $5.3(1.4-12.4)$ \\
\hline No & 303 & 3 & 1 & Reference & Reference \\
\hline \multicolumn{6}{|c|}{ Does the family eat from a common plate } \\
\hline Yes & 75 & 5 & 6.7 & 0.039 & $6.1(1.2-21.2)$ \\
\hline No & 304 & 3 & 1 & Reference & Reference \\
\hline \multicolumn{6}{|c|}{ Do you take locally prepared cold drinks } \\
\hline Yes & 50 & 5 & 10 & 0.014 & $6.9(1.4-32.3)$ \\
\hline No & 329 & 3 & 0.9 & Reference & Reference \\
\hline \multicolumn{6}{|c|}{ Does the family wash hands in common } \\
\hline \multicolumn{6}{|c|}{ basin } \\
\hline Yes & 57 & 5 & 8.8 & 0.022 & 7.3(1.9-31.2) \\
\hline No & 322 & 3 & 0.9 & Reference & Reference \\
\hline \multicolumn{6}{|c|}{ Monthly income } \\
\hline$<20,000.00$ & 256 & 8 & 2.5 & 0.025 & $0.2(0.03-0.8)$ \\
\hline$>20,001.00$ & 123 & 0 & 0 & & \\
\hline
\end{tabular}

OR - Odds ratio; CI - confidence interval; a - adjusted odds ratio; ND - Not done; P value- significant level

\section{DISCUSSION}

Epidemiological studies are essential in preventing and managing any disease/condition. This study, the first of its kind in Garissa county was a buildup of growing need for data on enteric fever given the lack of access to clean water, sanitation, proper housing and sufficient food in in this Semi-Arid Region of North Eastern Kenya (UNHABITAT, 2006). These attributes are essential risk factors for transmission of $S$. Typhi and $S$. Paratyphi. Specifically, this study determined the serotypes, antimicrobial resistance and associated factors this region of Kenya. In this study, $S$. Typhi and $S$. Paratyphi A and B were isolated for a total of $25 \%, 25 \%$ and $50 \%$ of entericfever patients respectively. Thus, the ratio of isolation of $S$. Typhi and S. Paratyphi A and B was 1: 1: 2 which was in contrary to other studies conducted globally which reported ratio from 1.6: 1 to 4: 1 (Bhattacharya et al., 2011; WHO, 2012). Though the incidence of $S$. Typhi remains high, several recent studies have highlighted the progressive increased proportion of S. Paratyphi A in the past decade (Dutta et al., 2014; Makkar et al., 2018).

Clinically, typhoid and paratyphoid fever are indistinguishable. Furthermore, many other acute febrile illnesses such as dengue, leptospirosis, and malaria may present a clinical picture similar to that of typhoid fever (Radhakrishnan et al., 2018). The results from this and other studies inevitably shows the importance for accurate and early diagnosis of typhoid and paratyphoid fever. The accurate diagnosis requires laboratory confirmation (Parry et al., 2011; Radhakrishnan et al., 2018). The development of practical, affordable, and accurate (i.e., both sensitive and specific) diagnostic tools is key to typhoid fever management and control.

In this study, attributes related to water and food (such as often eating outside homestead, family eating from a common plate, taking locally prepared cold drinks, family wash hands in common basin) were found associated with S. Typhi and S. Paratyphi infection. As it is expected, Garissa is a semi-arid area marked by water shortage and poor sanitation hygiene. The association of food and water related behavior is not surprising. It should be noted that we did not distinguish if these factors were specific to either S. Typhi or S. Paratyphi. Similar to our study many authors investigating enteric fever do not distinguish factors coincide to either typhoid or paratyphoid (Vollaard et al., 2004). The assumption is that in paratyphoid fever, a higher dose of bacteria is required for infection than in typhoid fever; consequently, food is implicated as the major vehicle for transmission of paratyphoid fever, since Salmonella bacteria can multiply in food (Vollaard et al., 2004). Undoubtedly, risk factors for both typhoid and paratyphoid fever have been identified in several epidemiologic studies suggesting either waterborne or foodborne transmission (Sur et al., 2007; Anand et al., 2010; Khan et al., 2012; Mogasale et al., 2018). The odds of typhoid fever among those exposed to unsafe water ranged from 1.06 to 9.26 (Mogasale et al., 2018). 
In our study, previous laboratory confirmation of typhoid was independently associated with being positive for enteric fever infection. According to WHO, (2017) Laboratory confirmation should always be sought for clinically suspected cases. Confirmation by culture (or validated molecular methods, as available) is essential as typhoid fever, paratyphoid fever and other invasive salmonellosis can present as a non-specific febrile illness, and current serological tests lack diagnostic specificity. It can be argued that those patients who sought laboratory confirmation had clinical signs and symbols for enteric fever. Confirmation is essential to assess the proportion of enteric fever caused by these different organisms, determine antimicrobial susceptibility and do molecular epidemiology studies (WHO, 2017)

Participant's low income was a key predictor for S. Typhi or S. Paratyphi infection. This was similar to other reports. Mogasale et al., (2018) besides water-related risk, identified other risk factors related to socioeconomic aspects, type of food consumption, knowledge and awareness about typhoid fever, and hygiene practices.

\section{Antibiotic susceptibility profiles of Salmonella strains}

In this study MDR Salmonella resistant was observed to ampicillin, tetracycline, gentamycin and chloramphenicol which is similar to different studies. Increase in the incidence of MDR Salmonella resistant to ampicillin, chloramphenicol, cotrimoxazole, streptomycin, furazolidone and tetracyclines is an emerging problem and a matter of concern worldwide (Dutta et al., 2014; Pakistan, 2016, Makkar et al., 2018). Encouragingly, in this study ciprofloxacin, nalidixic acid and trimethoprim-sulfamethoxazole were still effective against Salmonella strain. This is contrary to other studies such as in India Makkar et al., (2018), observed higher level of resistance to ciprofloxacin, co-trimoxazole, ampicillin, and third-generation cephalosporins. Most typhoid fever infections diagnosed in the United States are fluoroquinolone nonsusceptible. therefore, health care providers should not use fluoroquinolones as empiric therapy, especially in returning travelers from South Asia (Date et al., 2016). Fluoroquinolone nonsusceptibility has been associated with treatment failure or delayed clinical response (Crump et al., 2015). The emergence of fluoroquinolone nonsusceptible strains that are resistant to third-generation cephalosporins, such as ceftriaxone has been observed in Pakistan and other countries (Ryan and Andrews, 2018). In recent data from Pakistan published as part of the surveillance for enteric fever in Asia project (SEAP), over half of all S. Typhi isolates were multidrug resistant. Fluoroquinolone resistance was noted in nearly $90 \%$ of S. Typhi and S. Paratyphi isolates (Qamar et al., 2018). A longitudinal study of typhoid fever trends at three large hospitals in India showed a fall in resistance rates for ampicillin, chloramphenicol, and co-trimoxazole between 2000 and 2014, as resistance to more widely used antibiotics has risen (Balaji et al., 2018). Near universal resistance to ciprofloxacin has been observed in recent isolates from India (Dahiya et al., 2017). In our study, the patients with suspected severe or complicated typhoid fever might need to be treated with a fluoroquinolone and carbapenem and the treatment regimens can be adjusted when culture and sensitivity results are available.

our findings indicate the occurrence of enteric fever in this region facing climatic and economic hardship where majority of the populations have limited access to diagnostic services. This is an indication that the disease burden is poorly quantified and policy makers have lacked the data needed to make decisions about the deployment of enteric fever prevention measures and vaccines. Further, isolation of the antimicrobial resistance $S$. Typhi and $S$. Paratyphi in this study points strongly to need to establish hospital antimicrobial policy and antimicrobial prescribing guidelines. Periodic monitoring of the antibiogram pattern along with the implementation of strict antibiotic policies and patient education are crucial. Proper feeding, sanitation and hygiene practices are also likely to prevent and reduce the burdened on enteric fever in this region.

\section{Acknowledgments:}

We are grateful to the research participants who contributed greatly to this study. In addition, this study could not have been completed without the hard work and dedication of the research assistant in the field. Special thanks go to the supervisors for their support and input during various stages of this research project.

\section{Competing interests}

The authors declare no competing interests. 


\section{Authors' contributions}

This work was part of Master of Science degree for KSS in Laboratory Management and Epidemiology of Jomo Kenyatta University of Agriculture and Technology. KSS, ZN and SK conceived the study. KSS collected and analyzed the data and prepared the draft manuscript. ZN and SK provided guidance and mentorship during the implementation of the study. All authors reviewed and approved the final manuscript.

\section{Reference}

1. Anand P. K., Ramakrishnan R. Investigation of the outbreak of typhoid in a village of Thar Desert Rajasthan, India. Indian Journal of Medical Research. 2010;131(6):799-803.

2. Ao TT, Feasey NA, Gordon MA, Keddy KH, Angulo FJ, Crump JA. (2015) Global burden of invasive nontyphoidal Salmonella disease, 2010. Emerg Infect Dis. 2015;21(6):941-9 (https://wwwnc.cdc.gov/eid/article/21/6/14-0999 article).

3. Balaji V, Kapil A, Shastri J, et al. Longitudinal typhoid fever trends in India from 2000 to 2015. Am J Trop Med Hyg 2018;99(3_Suppl):34-40.

4. Bassiahi A.S, Mberu B, Elungata P, Lankoande B, Millogo R, Beguy D, \& Compaore Y. Understanding inequities in child vaccination rates among the urban poor: Evidence from Nairobi and Ouagadougou health and demographic surveillance systems. Journal of Urban Health. 2014; 92:1

5. Bhattacharya SS, Das U, Choudhury BK. Occurrence \& Antibiogram Salmonella Typhi \& S. Paratyphi A isolated from Rourkela, Orissa. Ind J Med Microbiol 2011; 133: 431-3.

6. Breiman RF, Cosmas L, Njuguna H, Audi A, Olack B, Ochieng JB, Wamola N, Bigogo GM, Awiti G, Tabu CW, Burke H, Williamson J, Oundo JO, Mintz ED, Feikin DR. Population-based incidence of typhoid fever in an urban informal settlement and a rural area in Kenya: implications for typhoid vaccine use in Africa. PLoS One. 2012;7(1):e29119. doi: 10.1371/journal.pone.0029119. Epub 2012 Jan 19.

7. CLSI. Performance Standards for Antimicrobial Susceptibility Testing. 27th ed. CLSI supplement M100. Wayne, PA: Clinical and Laboratory Standards Institute; 2017.

8. Crump JA, Sjölund-Karlsson M, Gordon MA, Parry CM. Epidemiology, clinical presentation, laboratory diagnosis, antimicrobial resistance, and antimicrobial management of invasive Salmonella infections. Clin Microbiol Rev 2015; 28:901-37.

9. Dahiya S, Sharma P, Kumari B, et al. Characterisation of antimicrobial resistance in Salmonellae during 2014-2015 from four centres across India: An ICMR antimicrobial resistance surveillance network report. Indian J Med Microbiol 2017; 35:61-8.

10. Date KA, Newton AE, Medalla F, et al. Changing patterns in enteric fever incidence and increasing antibiotic resistance of enteric fever isolates in the United States, 2008-2012. Clin Infect Dis 2016; 63:322-9.

11. Dutta S, Das S, Mitra U, et al. Antimicrobial Resistance, Virulence Pro- files and Molecular Subtypes of Salmonella enterica Serovars Typhi and Paratyphi A Blood Isolates from Kolkata, India during 2009-2013. PLoS ONE 2014; 9(8): e101347.

12. Dutta S, Das S, Mitra U, et al. Antimicrobial Resistance, Virulence Pro- files and Molecular Subtypes of Salmonella enterica Serovars Typhi and Paratyphi A Blood Isolates from Kolkata, India during 2009-2013. PLoS ONE 2014; 9(8): e101347

13. GBD 2017 Typhoid and Paratyphoid Collaborators. The global burden of typhoid and paratyphoid fevers: a systematic analysis for the Global Burden of Disease Study 2017. The Lancet Infectious Diseases. 18 Feb 2019. doi:10.1016/ S1473-3099(18)30685-6.

14. Harris JB, Brooks WA. Typhoid and paratyphoid (enteric) fever. In: Magill AJ, Ryan ET, Hill DR, Solomon T, eds. Hunter's tropical medicine and emerging infectious diseases, 9th edn. Philadelphia, PA: Elsevier, 2013: 568-76.

15. Kariuki S, Revathi G, Kiiru J, Mengo DM, Mwituria J, Muyodi J, Munyalo A, Teo Y, Holt K, Kingsley $\mathrm{R}$, and Dougan G. Typhoid in Kenya Is Associated with a Dominant Multidrug-Resistant Salmonella enterica Serovar Typhi Haplotype That Is Also Widespread in Southeast Asia. Journal of Clinical Microbiology. 2010. 2171-2176

16. Kariuki, S., G. Revathi, J. Muyodi, J. Mwituria, A. Munyalo, S. Mirza, and C. A. Hart. 2004. Characterization of multidrug-resistant typhoid outbreaks in Kenya. J. Clin. Microbiol. 42:1477-1482.

17. Kariuki, S., G. Revathi, N. Kariuki, J. Kiiru, J. Mwituria, and C. A. Hart. 2006. Characterization of community acquired non-typhoidal Salmonella from bacteraemia and diarrhoeal infections in children admitted to hospital in Nairobi, Kenya. BMC Microbiol. 6:101. 
18. Kebede A, Kemal J, Alemayehu H, Habte Mariam S. Isolation, Identification, and Antibiotic Susceptibility Testing of Salmonella from Slaughtered Bovines and Ovines in Addis Ababa Abattoir Enterprise, Ethiopia: A Cross-Sectional Study. Int J Bacteriol. 2016:3714785.

19. Khan M. I., Ochiai R. L., Soofi S. B., et al. Risk factors associated with typhoid fever in children aged 2-16 years in Karachi, Pakistan. Epidemiology and Infection. 2012;140(4):665-672.

20. Kimani-Murage EW, Fotso JC, Egondi T, Abuya B, Elungata P, Ziraba AK et al. Trends in childhood mortality in Kenya: the urban advantage has seemingly been wiped out. Health Place. 2014; 29:95- 103

21. Lemeshow, S., Hosmer, D. K., Klar, J. \& Lwanga, S. K. 1990. World Health Organization. Adequacy of samples size in health studies. www.tbrieder.org/publications/books_english/ lemeshow_samplesize.pdf (accessed February 2017).

22. Makkar A, Gupta S, Khan I, Gupta R, Rajmohan KS, Chopra H, Gupta M, Bansal S, Poonia B, Malik M, Panda P. Epidemiological Profile and Antimicrobial Resistance Pattern of Enteric Fever in a Tertiary Care Hospital of North India - a Seven Year Ambispective Study. Acta Medica (Hradec Králové) 2018; 61(4): 125-130

23. Mogasale VV, Ramani E, Mogasale V, Park JY, Wierzba TF. Estimating Typhoid Fever Risk Associated with Lack of Access to Safe Water: A Systematic Literature Review. J Environ Public Health. 2018; 2018:9589208.

24. Parry CM, Wijedoru L, Arjyal A, Baker S, 2011. The utility of di- agnostic tests for enteric fever in endemic locations. Expert Rev Anti Infect Ther 9: 711-725.

25. Phan, M. D., C. Kidgell, S. Nair, K. E. Holt, A. K. Turner, J. Hinds, P. Butcher, F. J. Cooke, N. R. Thomson, R. Titball, Z. A. Bhutta, R. Hasan, G. Dougan, and J. Wain. 2009. Variation in Salmonella enterica serovar Typhi IncHI1 plasmids during the global spread of resistant typhoid fever. Antimicrob. Agents Chemother. 53:716-727

26. Qamar FN, Yousafzai MT, Sultana S, et al. A retrospective study of laboratory-based enteric fever surveillance, Pakistan, 2012-2014. J Infect Dis 2018;218(suppl_4): S201-5. doi:10.1093/ infdis/jiy205

27. Radhakrishnan A, Als D, Mintz E, Crump J, Stanaway J, Breiman R, Bhutta Z. Introductory Article on Global Burden and Epidemiology of Typhoid Fever. Am. J. Trop. Med. Hyg., 99(Suppl 3), 2018, pp. 4 9

28. Ryan ET, Andrews J. Treatment and prevention of enteric (typhoid and paratyphoid) fever. UpToDate 2018. https://www.uptodate.com/ contents/treatment-and-prevention-of-enteric-typhoid-andparatyphoid-fever?topicRef $=2708 \&$ source=related_link

29. Sur D., Ali M., Von Seidlein L., et al. Comparisons of predictors for typhoid and paratyphoid fever in Kolkata, India. BMC Public Health. 2007;7, article no. 289 doi: 10.1186/1471-2458-7-289.

30. UN-HABITAT. The State of the World's Cities Report 2006/2007. London: United Nations Human Settlements Programme; 2006.

31. Vollaard AM, Ali S, van Asten HAGH, et al. Risk Factors for Typhoid and Paratyphoid Fever in Jakarta, Indonesia. JAMA. 2004;291(21):2607-2615. doi:10.1001/jama.291.21.2607

32. Whitaker JA, Franco-Paredes C, del Rio C, Edupuganti S. Rethinking typhoid fever vaccines: implications for travelers and people living in highly endemic areas. J Travel Med 2009; 16:46-52.

33. World Health Organization: Indian Network for Surveillance of Antimicrobial Resistance. Antibiogram of Salmonella enterica serovar Typhi and Salmonella enterica serovar Paratyphi A: a multi-centre study from India. WHO South-East Asia J Public Health 2012; 1: 182-8.

34. World Health Organization. Typhoid vaccines: WHO position paper - March 2018. Wkly Epidemiol Rec. Wkly Epidemol Rec. 2018;93(13):153-72.

35. World Health Organization. Vaccination in acute humanitarian emergencies: a framework for decision making. Geneva: World Health Organization; 2017 (http://apps.who.int/iris/bitstream/10665/255575/1/WHO-IVB-17.03-eng.pdf). 